\title{
Establishing a daily rainfall occurrence simulation model for the Langat River catchment, Malaysia
}

\author{
Chau Yuan Lian, Yuk Feng Huang*(D) and Lloyd Ling \\ Department of Civil Engineering, Lee Kong Chian Faculty of Engineering and Science, Universiti Tunku Abdul \\ Rahman, Bandar Sungai Long, Cheras, 43000 Kajang, Selangor, Malaysia. \\ *Corresponding author. e-mail: huangyf@utar.edu.my
}

MS received 10 October 2017; revised 26 April 2018; accepted 8 May 2018; published online 25 January 2019

For the study of water resources of a catchment, an immediate task would be to establish a good model for predicting the probable daily rainfall occurrence and rainfall amount. This study presents the simulation of daily rainfall occurrence using the generalized linear model (GLM), the non-homogeneous hidden Markov model (NHMM) and the bootstrap aggregated classification tree (BACT) model. The major challenge of NHMM is the determination of optimum number of hidden states, which can be achieved using the Bayesian information criterion score. While the determination of number of grown tree is another challenge for BACT model, this critical task can be achieved with the help of out-ofbag classification error. Both the NHMM and BACT model outperformed the GLM to capture the rainfall persistence and spell lengths distribution. Through the validation phase, the BACT model exhibited better performance with the higher indices of probability of detection, critical success index, Heidke skill score and Peirce skill score, than other models. The prediction ability of the NHMM is equivalent to an unskilled random forecast with the skill scores nearly equal to zero. At the end, the BACT model was recommended as the appropriate daily rainfall occurrence model for this study.

Keywords. Statistical downscaling; generalized linear model; non-homogeneous hidden Markov model; bootstrap aggregated classification tree; daily rainfall occurrence model; Langat River catchment.

\section{Introduction}

Climate change due to rising of greenhouse gas (GHG) concentration retained in atmosphere is the most important issue of global concern. The frequent occurrence of extreme hydrological events, a result of climate change, can bring about significant impacts on the productivity of crops, occurrence of floods and droughts, supply of water resources and many calamities that has become daily occurrences around the world. The climate change impact on the physical and biological systems on the earth are anticipated to extend to our future (Sullivan and Huntingford 2009).

Under the consideration of future climate change, global climate models (GCMs), which consider the atmospheric forcing under different scenarios, are available to predict the global future rainfall series. However, the outputs of these simulations from the GCMs in coarse grid, are not recommended for direct use in a regional or locality grid. Therefore, a downscaling technique is required to reduce the inherent discrepancies and acquire the prerequisite local climate variables. Statistical downscaling is 
defined as a statistical technique that is used to acquire climate variables at the required temporal and spatial resolution, by relating the large-scale or global climate variables to regional or local variables refined for further use in climate change impact studies (Buishand et al. 2004).

There are three most common statistical downscaling techniques, such as regression-based techniques, weather pattern classification and weather generators. The well-known regression-based models include artificial neural network (Schoof and Pryor 2001; Ahmed et al. 2015) and statistical downscaling model (SDSM) (Wilby and Dawson 2013; Singh et al. 2015) are commonly used to establish a relationship between the predictors and predictand for downscaling purposes. Rainfall occurrence can be denoted by binary sequence, where one represents wet day and zero represents dry day (Beckmann and AdriBuishand 2002). Logistic regression, which is a particular class of generalized linear model (GLM), has been widely used in simulating the rainfall occurrence. The use of maximum likelihood methods is the most important characteristic in the GLM, which allows the response variables and error terms from the fitted models to have different types of distribution other than the normal distribution. In addition, the logit link of the GLM is the common link to be used in hydrologic models, because it is applicable to binary data and logistic regression. The approach of logistic regression in the GLM is required when the predicted value is in binary form and this approach is capable of generating the probability in between zero and one. Several studies showed the good simulation performance of GLM in modelling the rainfall occurrence based on a range of atmospheric variables (Fealy and Sweeney 2007; Lee et al. 2011; Kenabatho et al. 2012).

The approach of weather classification is to group the local variables with respect to different classes of global climate variables. The non-homogeneous hidden Markov model (NHMM) (Kioutsioukis et al. 2008; Mares et al. 2014) is able to capture the spatial and temporal variability of rainfall through the recognition of noticeable weather patterns in the multi-station region. In addition, the NHMM has been proven in the studies of Robertson et al. $(2004,2009)$ to be able to capture the characteristic of daily rainfall occurrence, in terms of spell lengths and convey the majority of the GCMs' large-scale inter-annual simulation skill to the daily rainfall sequence. In China, Liu et al. (2011) showed that the NHMM performed better than SDSM in simulating the daily rainfall data. Following these studies, NHMM has been widely used as a downscaling model over the years, for simulating the rainfall projections (Greene et al. 2011; Fu et al. 2013; Liu et al. 2013).

Classification is one of the supervised machine learning techniques, which can be used to predict the value from any input data, after learning the relationship between pairs of input and target output data. Classification and Regression Tree (CART) is a model that refers to both classification and regression trees, which has been widely used in predicting the rainfall occurrence and amount as a function of course resolution atmospheric variables. Based on present studies, the occurrence of daily rainfall state was first generated using an unsupervised data classification technique, namely K-means clustering coupled with a CART model. Consequently, the generation of daily rainfall amount conditioned on the rainfall state was done by a nonparametric kernel regression model (Kannan and Ghosh 2013 ; Salvi et al. 2013) and a beta regression model (Mandal et al. 2016). Both models showed the abilities in capturing the spatial and temporal variability of rainfall.

Nevertheless, bootstrap aggregation (bagging) (Gaitan et al. 2014) and Random Forests (RF) are the ensemble machine learning techniques designed to improve the weak prediction of a single classification tree. The RF is generated based on the CART technique and deemed as an improvement over bagged tree due to its ability of yielding high prediction accuracy and being robust against overfitting problem. Jing et al. (2016) downscaled the monthly rainfall data over north China using knearest neighbour (k-NN), support vector machine (SVM), CART and RF. They found that SVM and RF-based models performed better than other models, but the good performance of SVM model was strongly dependent on the residual correction. In recent studies, RF-based model was also employed to perform the spatial downscaling of monthly rainfall (Shi and Song 2015; He et al. 2016). In addition, there are few recent studies that (Chau and Wu 2010; Wu et al. 2010) employed the data pre-processing approaches to improve the rainfall prediction of their proposed model.

Prior to the future downscaling stage, the simulation result of a developed weather generator is very important. The high accuracy of a model in simulating the observed weather data indicates that the relationship between large-scale 
atmospheric variables and local weather data are highly correlated by the model, thus the downscaled future weather data turns out to be more reliable for the use in climate change impact studies. It is important to establish the best rainfall amount prediction model of a catchment and for many purposes, on a daily scale basis. A priori to this is the need to establish a reliable daily rainfall occurrence simulation model. The honing combination of these two models would be desirous for managing the water resources of a catchment. For example, a stochastic model was developed in the study of $\mathrm{Ng}$ et al. (2017), to simulate the rainfall occurrence, followed by an intensity model to simulate the rainfall characteristics in Kelantan River Basin. This study covers the determination of an appropriate daily rainfall occurrence model in the tropical Langat River catchment using the generalized linear model (GLM), the non-homogeneous hidden Markov model (NHMM) or the bootstrap aggregated classification tree (BACT) model. The Langat River catchment is situated in a tropical rainforest country, and the surrounding area of the catchment undergoes rapid urbanization, especially the land-use of the catchment, which has been converted from agricultural to industrial (Memarian et al. 2014; Saudi et al. 2015). The rapid urban development and population growth definitely increase the rate of surface runoff, resulting in the occurrence of flash floods. Other than the water supply, the two main reservoirs within the catchment are also used for flood mitigation purposes. Therefore, the ability of a model to downscale the rainfall series is very important for a catchment. There were two major challenges in this study during the process of developing the NHMM and BACT model. For the determination of hidden states in NHMM, it was achieved using the computed Bayesian Information Criterion (BIC) score of each state. While, the determination of grown trees in BACT model was solved using the prediction error generated from out-of-bag sample. The second goal of this study is to compare the rainfall occurrence models based on their performance in terms of rainfall persistence, spell lengths distribution and matching. At the end, an appropriate rainfall occurrence model is to be selected to simulate the daily rainfall occurrence at the tropical Langat River catchment, setting the background for the follow-up establishment of an appropriate daily rainfall amount simulation model. Besides, the selected rainfall occurrence model can be proceeded further to downscale the future rainfall occurrence of basin under different emission scenarios, using the output of GCMs. The high accuracy of downscaled data from the selected model is relatively important for an effective planning and management of water resources and increase the awareness of flood occurrence within the catchment.

\section{Study area}

The Langat River catchment, situated at the south of the Klang Valley, has been chosen as the study area for this research. This catchment is within the latitudes $2^{\circ} 40^{\prime} 152^{\prime \prime}-3^{\circ} 16^{\prime} 15^{\prime \prime} \mathrm{N}$ and longtidues $101^{\circ} 19^{\prime} 20^{\prime \prime}-102^{\circ} 1^{\prime} 10^{\prime \prime} \mathrm{E}$, with the total area of $\sim 2282 \mathrm{~km}^{2}$. There are two main reservoirs within the catchment, namely the old Langat reservoir and the new Semenyih reservoir with catchment areas of 54 and $41 \mathrm{~km}^{2}$, respectively. The main fuction of both reservoirs is to supply water for industrial and domestic uses, for the areas that include Cheras, Hulu Langat, Kuala Langat, Petaling, Putrajaya and Sepang. In addition, Putrajaya is a planned city and federal adminitrative centre of Malaysia with dense population. The Langat reservoir also serves a hydro-power plant, that supplies electricity in moderate capacity for the population within Langat Valley.

\section{Data}

\subsection{Observed rainfall series}

Four rainfall stations (numbered as 2815001, 2917001, 2913001 and 3118102) were chosen and their daily observed rainfall series were acquired from the Department of Irrigation and Drainage Malaysia (DID) and the Malaysian Meteorological Department (MMD). In order to achieve the accurate and reliable downscaling results, the minimum requirement for the duration of observed rainfall series is 30 yrs. These four stations recorded the daily rainfall series from 1975 to 2012, which meet the minimum requirement period for modelling. In this study, a threshold value of $0.1 \mathrm{~mm}$ was used to define a day as dry or wet; a larger threshold value may cause the underestimation of rainfall occurrence in Langat River catchment. The detailed descriptions of stations are provided in table 1 and they are located within the Langat River catchment as illustrated in figure 1. 
Table 1. List of respective station code, name, study period, longitude and latitude.

\begin{tabular}{llcrr}
\hline Station code & \multicolumn{1}{c}{ Station name } & Study period & Longitude & Latitude \\
\hline 2815001 & Pejabat JPS Sg. Manggis & $1975-2012$ & $101^{\circ} 32^{\prime} \mathrm{E}$ & $2^{\circ} 49^{\prime} \mathrm{N}$ \\
2913001 & P/KWLN/S Telok Gong & $1975-2012$ & $101^{\circ} 23^{\prime} \mathrm{E}$ & $2^{\circ} 55^{\prime} \mathrm{N}$ \\
2917001 & RTM Kajang & $1975-2012$ & $101^{\circ} 47^{\prime} \mathrm{E}$ & $2^{\circ} 59^{\prime} \mathrm{N}$ \\
3118102 & Sek. Keb. Kg. Sg. Lui & $1975-2012$ & $101^{\circ} 52^{\prime} \mathrm{E}$ & $3^{\circ} 10^{\prime} \mathrm{N}$ \\
\hline
\end{tabular}

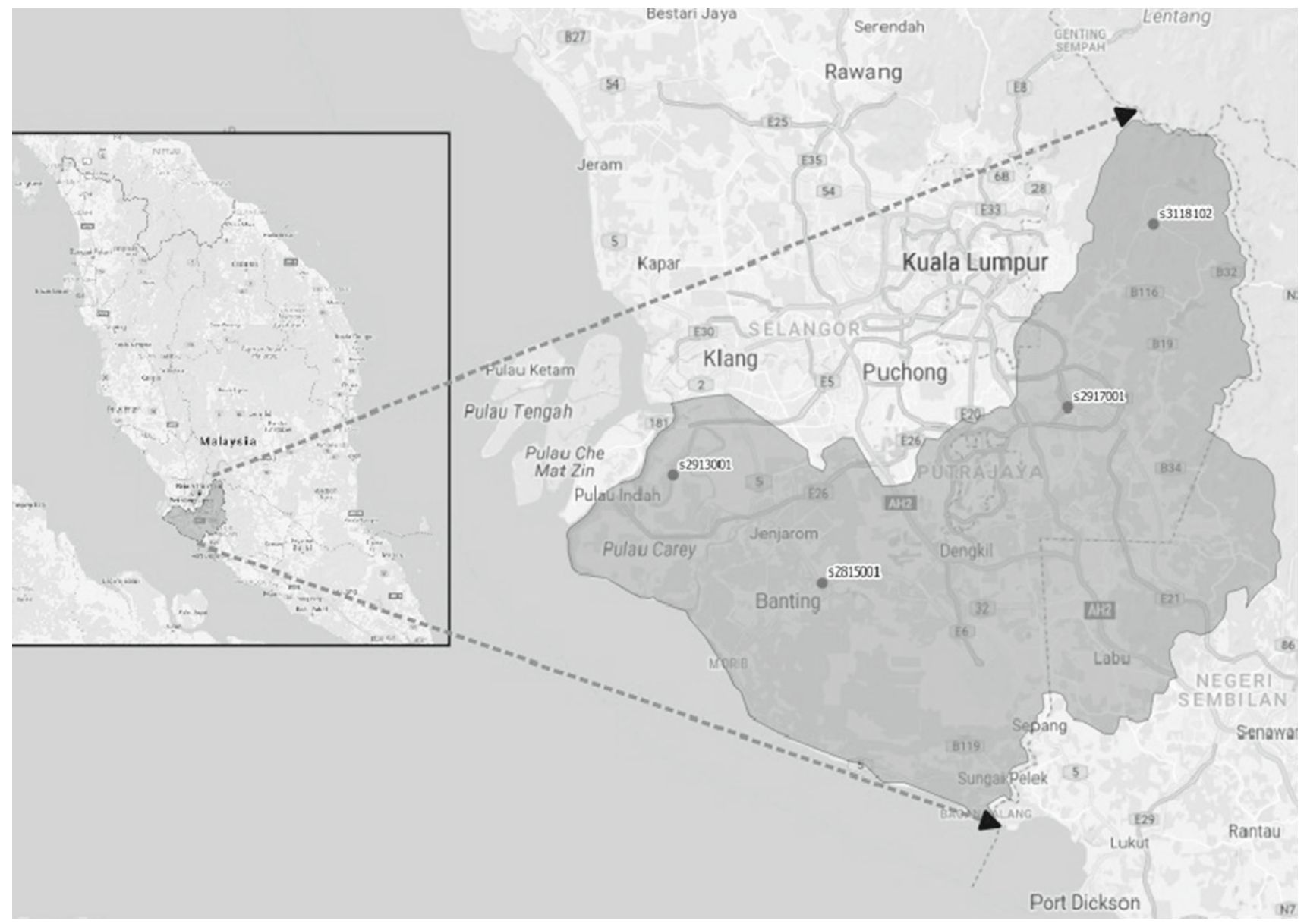

Figure 1. Location of the selected rainfall stations at the Langat River catchment.

\subsection{Reanalysis dataset for atmospheric predictors}

The predictor variables used in this study were from the large-scale re-analysis datasets obtained from the National Centre for Environmental Prediction and National Center for Atmospheric Research (NCEP\&NCAR). These potential predictors are the daily values of 26 variables (1961$2005)$, which comprise of not only the circulation variables (i.e., geopotential and wind component), but also temperature, radiation and moisture variables (specific humidity), etc. Due to the inconsistent periods between observed rainfall series and reanalysis dataset, the data from 1976 to 2005 were extracted out from their respective raw dataset for modelling. Also, each variable underwent the lagtransformation process (lag -9 to 9 ) for the purpose of selecting the suitable predictor variables, which are highly correlated with the observed rainfall series, for each station.

\section{Methodology}

\subsection{Screening of predictors}

A complete set of potential predictors contains plenty of information and almost all of them are mutually correlated, but some of them may be treated as excessive information since they cannot 
provide any significant effect in downscaling process. Therefore, the correlation between rainfall and potential predictor, which can be expressed by explained variance, are significant during the selection of suitable predictors. In addition, the association between potential predictors themselves should be considered and measured using the partial correlation analysis. In this study, each variable and its lag-transformed variables were screened with the daily observed rainfall series for selecting the most suitable set of predictors for each station, under the consideration of explained variance and partial correlation analysis as selection criteria.

\subsection{Rainfall occurrence models}

\subsubsection{Generalized linear model (GLM)}

In this study, the GLM with logistic regression was employed to model the rainfall occurrence with binomial distribution in the MATLAB platform using the code of 'glmfit(binomial)' and 'glmval(logit)'. There are several distribution parameters that can be chosen form GLM, such as normal, gamma, Poisson and binomial. However, the binomial distribution was deemed to be suitable for this study because the response variable (observed rainfall occurrence series) is in binary form. The occurrence of wet and dry days can be represented as a binary series $\left\{y_{t}\right\}, y_{t}=1$, if day $t$ is wet and $y_{t}=0$, if day $t$ is dry. The key parameter in the logistic model is the probability $P$ of a day being wet. Let $P_{i}$ denotes the probability for the $i$ th case in the dataset conditional on a vector $X_{i}^{\prime}$, then the rainfall occurrence is given by:

$$
\ln \left(\frac{P_{i}}{1-P_{i}}\right)=X_{i}^{\prime} \beta
$$

Equation (1) can be revised in respect to odds as:

$$
\left(\frac{P_{i}}{1-P_{i}}\right)=\exp ^{X_{i}^{\prime} \beta},
$$

where $\exp =$ base of the natural logarithms and $\beta=$ estimated coefficients.

The selection of parameters in the logistic regression is achieved using the maximum likelihood estimation. This approach helps to determine the parameters which can maximize the likelihood function to produce the similar targeted output. In addition, there are several link functions that can be chosen to map between the mean and linear predictors, namely identity (default for normal distribution), log (default for Poisson distribution), logit (default for binomial distribution), probit and reciprocal (default for gamma distribution) links. The logit link was adopted in this study as it ensures the predicted value lies in the interval between zero and one, where one is interpreted as wet days. The threshold value for the probability of predicted value is set as 0.5 . In other words, the day with the probability smaller than 0.5 was marked as zero to represent a dry day and the day with the probability greater than 0.5 was marked as one to represent a wet day.

\subsubsection{Non-homogeneous hidden Markov model (NHMM)}

In this study, the NHMM were performed using an available software toolkit, namely the multivariate non-homogeneous hidden Markov model toolbox (MVNHMM). The NHMM is the modification of hidden Markov model (HMM) by including the exogenous atmospheric predictors for rainfall simulation. Firstly, the observed daily rainfall occurrence series were fitted into NHMM for determining the optimum hidden state. The rainfall distribution type was set to independent and Bernoulli in advanced options within the software, due to the rainfall at each station is independent of other stations and the data used is binary data. There are two conditional independence assumptions for the rainfall data. The first assumption is that the multivariate rainfall observations $R_{t}$ at time $t$ are independent of all other variables in the model up to time $t$, conditional on the weather state $S_{t}$ at time $t$ as:

$$
P\left(R_{t} \mid S_{1: t}, R_{1: t-1}\right)=P\left(R_{t} \mid S_{t}\right)
$$

The second assumption is that the hidden state process, $S_{1: t}$ is the first-order Markov process

$$
P\left(S_{t} \mid S_{1: t-1}\right)=P\left(S_{t} \mid S_{t-1}\right) .
$$

And this first-order Markov process is homogeneous in time, which means the transition probability matrix of the states does not change over time.

After the number of optimum hidden state is determined, the selected predictors are introduced into the NHMM. Let $X_{t}$ be a D-dimensional column vector of predictors for day $t$, and $X_{1: T}$ 
denotes the sequence of $X_{1}, \ldots, X_{T}$. Equation (4) is then replaced with

$$
P\left(S_{t} \mid S_{1: t-1}, X_{1: T}\right)=P\left(S_{t} \mid S_{t-1}, X_{t}\right) .
$$

Therefore, the hidden state on the day $t$ depends on both predictor vector $X_{t}$ for day $t$ and the value of the hidden state $S_{t-1}$ on day $t-1$. Since $X_{t}$ can vary in time, the transition probabilities between states also differ with respect to time in response to changes in $X$, thus it is so called non-homogeneous model. The NHMM was fitted with the hidden states from one to seven and the Bayesian information criterion (BIC) score was used as the guideline in selecting the most appropriate number of hidden states.

In this study, the iterative expectation maximization (EM) algorithm was used to estimate the parameter of NHMM. However, the limitation of EM algorithm is that it can be trapped at local maxima and consequently fails to reach the global maxima. To avoid the high possibility of EM algorithm converges to local maxima, this algorithm was initialized 10 times from different random starting point, with the EM run to convergence for each starting point, and the parameter vector with maximum likelihood over all 10 runs was selected. There was a numerical test using a larger number of random starting points and it showed that the use of 10 initializations provide a good settlement between computation time and estimation of global maximum value (Pineda and Willems 2016). Instead of using delta-exponential with one component or two components, the rainfall amount were modelled using a delta-gamma model in this study. The main reason is because the delta-gamma model achieved the smaller BIC score than other two models at each station, as shown in table 8 . Thereafter, the rainfall occurrence series of the NHMM was visualized based on its simulated rainfall amount series.

\subsubsection{Bootstrap aggregated classification tree (BACT) model}

Decision trees are a learning technique, which is applicable to both classification and regression problems, and hence they are commonly referred as the classification and regression tree (CART). The approach of the CART model is to perform accurate classification or prediction based on the if-then logical conditions. A decision tree is formed using a binary recursive partitioning algorithm, which is an iterative data-splitting process, to split the data into child nodes with certain conditions and repeat itself until certain rules are met or no further splitting is possible (Kannan and Ghosh 2011).

In this study, the classification decision tree was selected to model the rainfall occurrence instead of using the regression decision tree. The rainfall occurrence state could be treated as a classification type problem, with the condition of predicting the value of response variables (rainfall state) from a combination of predictor variables. However, the bootstrap aggregation (bagging) of classification trees was employed in this study to improve the generalization of individual classification decision tree. The bootstrap aggregated classification tree model was developed in the MATLAB platform using the code of 'TreeBagger', where every tree in the ensemble is grown on an independently drawn bootstrap replica of input data. During the process of bootstrap aggregation, the number of predictor variables to be selected randomly for each node ('NVarToSample') was set to 'all' to invoke the random forest algorithm, which can provide high prediction accuracy and stability in model's performance. The random forest is similar to bagging but with an additional randomness (Breiman 2001). This algorithm was further explained by Liaw and Wiener (2002) in their study.

In addition, there were a few parameters set in the process of building the model. For instance, the 'OOBpred' was set to 'on' for determining the optimum number of grown tree in the BACT model. The priorities for each class ('prior') was set to 'uniform', where all class probabilities are equal. The nodes were split ('splitcriterion') based on their impurity using the code of 'deviance' for maximum deviance reduction.

\subsection{Performance measure of rainfall occurrence model}

In this study, the simulation performance of rainfall occurrence models were compared and evaluated in terms of rainfall persistence and spell lengths distribution. In addition, the probability of detection (POD), false alarm rate (FAR), critical success index (CSI), Heidke skill score (HSS) and Peirce skill score (PSS), were applied to further check the matching between the simulated and observed wet days. These indices were obtained from a $2 \times 2$ contingency table, as shown in table 2 with the following equations 


$$
P O D=\frac{a}{a+c}
$$

The $P O D$ ranges from zero to one with the perfect score of one and is sensitive to hits, but ignore the false alarms.

$$
F A R=\frac{b}{a+b}
$$

The FAR ranges from zero to one with the perfect score of one and is sensitive to false alarms, but ignore the misses.

$$
C S I=\frac{a}{a+b+c}
$$

The $C S I$ ranges from zero to one with $C S I=$ 0 indicating no wet day forecasts are matched and $C S I=1$ indicates all wet days forecasts are matched. $C S I$ is sensitive to hits and penalises both misses and false alarms.

$$
H S S=\frac{2(a d-b c)}{(a+c)(c+d)+(a+b)(b+d)} .
$$

The HSS ranges from negative one to one with $H S S=1$ indicating the perfect forecast and $H S S=0$ indicates the forecast is unskilled random forecast. If $H S S<1$, the forecast is worse and standard forecast is more accurate than forecast.

$$
P S S=\frac{a d-b c}{(a+c)(b+d)} .
$$

The $P S S$ ranges from negative one to one with $P S S>0$, whence then the number of hits exceeds the false alarms and the forecast has some skill, and

\begin{tabular}{|c|c|c|c|c|}
\hline & & \multicolumn{2}{|c|}{ Observed } & \\
\hline & & Yes & No & \\
\hline \multirow{2}{*}{ Forecast } & Yes & $a$ & $b$ & $a+b$ \\
\hline & No & $\begin{array}{c}c \\
a+c\end{array}$ & $\begin{array}{c}d \\
b+d\end{array}$ & $\begin{array}{c}c+d \\
n=a+b+c+d\end{array}$ \\
\hline
\end{tabular}
with $P S S \leq 0$ indicating the forecast is unskilled. Values of $a, b, c$, and $d$ are as defined in table 2 .

Table 2. Contingency table for possible outcomes of the rainfall occurrence models.

Four possible outcomes: (1) Number of events which are forecasted and actually occurred (a); (2) Number of events which are forecasted, but actually not occurred (b); (3) Number of events which are not forecasted, but actually occurred (c); (4) Number of events which are not forecasted and actually not occurred (d).

\section{Results and discussion}

\subsection{Predictors selection}

The predictors for each station were selected based on their explained variance with observed rainfall series and partial correlation analysis within predictors themselves. The explained variance describes the correlation between predictors and observed rainfall series, which means the higher explained variance value indicates that the predictor has higher correlation with observed rainfall series. However, same variables tend to be selected based on their explained variance values only, and this may affect the performance of the model. Hence, the partial correlation analysis between the predictors was also taken into account during the predictors selection process, for the purpose of avoiding this situation and selecting the suitable combination of different variables. Table 3 shows the selected predictors, which are suitable and highly correlated to observed rainfall at each station.

\subsection{Determination of hidden states in NHMM}

The determination of optimum number of hidden states is the major challenge in developing the NHMM for rainfall downscaling. This is because the number of hidden states provided to the NHMM must be sufficient to express the rainfall state of observed rainfall series in order to achieve the high accuracy of performance. However, the difficultly of this critical task can be reduced using the BIC as the selection criteria. With the use of BIC score, the optimum number of hidden state can be determined in an easier way. The quality of the fitted NHMM as a function of different number of hidden states were evaluated in terms of their computed BIC scores, as shown in table 4. For stations 2815001 and 2913001, it was found that the computed BIC scores reached their minimum points at three hidden states, which indicated that three hidden states were deemed to be sufficient in capturing the daily rainfall occurrence of these two stations. However, only two hidden states were required to capture the daily rainfall occurrence at stations 2917001 and 3118102, respectively.

\subsection{Determination of number of grown trees in $B A C T$ model}

The major challenge during the process of developing a BACT model is to determine the optimum 
Table 3. Description and lag-transformation of selected predictors that are highly correlated with the observed rainfall series at each station.

\begin{tabular}{|c|c|c|c|}
\hline Station & Predictors & Description & Lag \\
\hline \multirow[t]{5}{*}{2815001} & p8_fgl & Geostrophic airflow velocity at 850 hpa height & 2 \\
\hline & p8_ugl & Zonal velocity component at $850 \mathrm{hpa}$ height & 0 \\
\hline & s500gl & Specific humidity at 500 hpa height & 1 \\
\hline & s850gl & Specific humidity at 850 hpa height & -1 \\
\hline & shumgl & Near surface specific humidity & 0 \\
\hline \multirow[t]{4}{*}{2913001} & p8_ugl & Zonal velocity component at $850 \mathrm{hpa}$ height & -2 \\
\hline & p8_zgl & Vorticity at 850 hpa height & 1 \\
\hline & s500gl & Specific humidity at 500 hpa height & 1 \\
\hline & shumgl & Near surface specific humidity & -1 \\
\hline \multirow[t]{5}{*}{2917001} & p1_vgl & Meridional velocity component & 0 \\
\hline & p5_ugl & Zonal velocity component at $500 \mathrm{hpa}$ height & 0 \\
\hline & p8_fgl & Geostrophic airflow velocity at 850 hpa height & -1 \\
\hline & p8_zgl & Vorticity at 850 hpa height & 1 \\
\hline & shumgl & Near surface specific humidity & -1 \\
\hline \multirow[t]{5}{*}{3118102} & mslpgl & Mean sea level pressure & 2 \\
\hline & p5_ugl & Zonal velocity component at $500 \mathrm{hpa}$ height & 0 \\
\hline & p8zhgl & Divergence at $850 \mathrm{hpa}$ height & 0 \\
\hline & s850gl & Specific humidity at 850 hpa height & 0 \\
\hline & shumgl & Near surface specific humidity & 0 \\
\hline
\end{tabular}

Table 4. Bayesian information criterion (BIC) scores of NHMM as a function of different hidden sates at each station.

\begin{tabular}{lcccc}
\hline Hidden & \multicolumn{4}{c}{ BIC score } \\
\cline { 2 - 5 } state & 2815001 & 2913001 & 2917001 & 3118102 \\
\hline 1 & 9561.39 & 9567.06 & 10031.97 & 9926.01 \\
2 & 8742.39 & 8634.73 & $\mathbf{9 3 7 2 . 4 6}$ & $\mathbf{8 4 0 8 . 6 5}$ \\
3 & $\mathbf{8 6 8 2 . 4 3}$ & $\mathbf{8 5 1 2 . 5 3}$ & 9417.23 & 8530.30 \\
4 & 8747.36 & 8578.35 & 9444.62 & 8562.39 \\
5 & 8831.61 & 8667.51 & 9517.87 & 8573.03 \\
6 & 8939.37 & 8769.03 & 9606.28 & 8806.49 \\
7 & 9061.54 & 8895.14 & 9748.48 & 8843.79 \\
\hline
\end{tabular}

Note: Bold values indicate the minimum BIC score at each station

number trees to be grown in the model for learning the training data. This critical task can be done using the out-of-bag classification error. Out-ofbag (OOB) samples are defined as the data that were not included during the splitting process of each bootstrap sample. The prediction strength of BACT model can be measured based on the average prediction error generated by each OOB sample over the total grown trees in model. Basically, if a sufficient large number of trees is given, the determination of number of trees is considered to be less critical. This is because there is no further substantial reduction of error to be achieved after reaching the optimum number of grown tree in the model. In this study, 1000 trees were first used to determine the optimum number of grown tree in BACT model based on the generated classification error. As illustrated in figure 2, the OOB classification error decreased with the number of grown trees. However, in considering the computational efficiency and stability of prediction, the number of grown trees was set to 150 in BACT model for each station. This is because the OOB classification error is stabilized after 150 trees at each station and adding more trees is unnecessary as this will only cause the intensive computational time of model.

\subsection{Performance measure of rainfall occurrence models}

The rainfall occurrence models (GLM, NHMM and BACT model) of each station have been calibrated and validated using the daily rainfall occurrence series and selected predictors with a 20-years (1976-1995) and a 10-years (1996-2005) periods, respectively. 

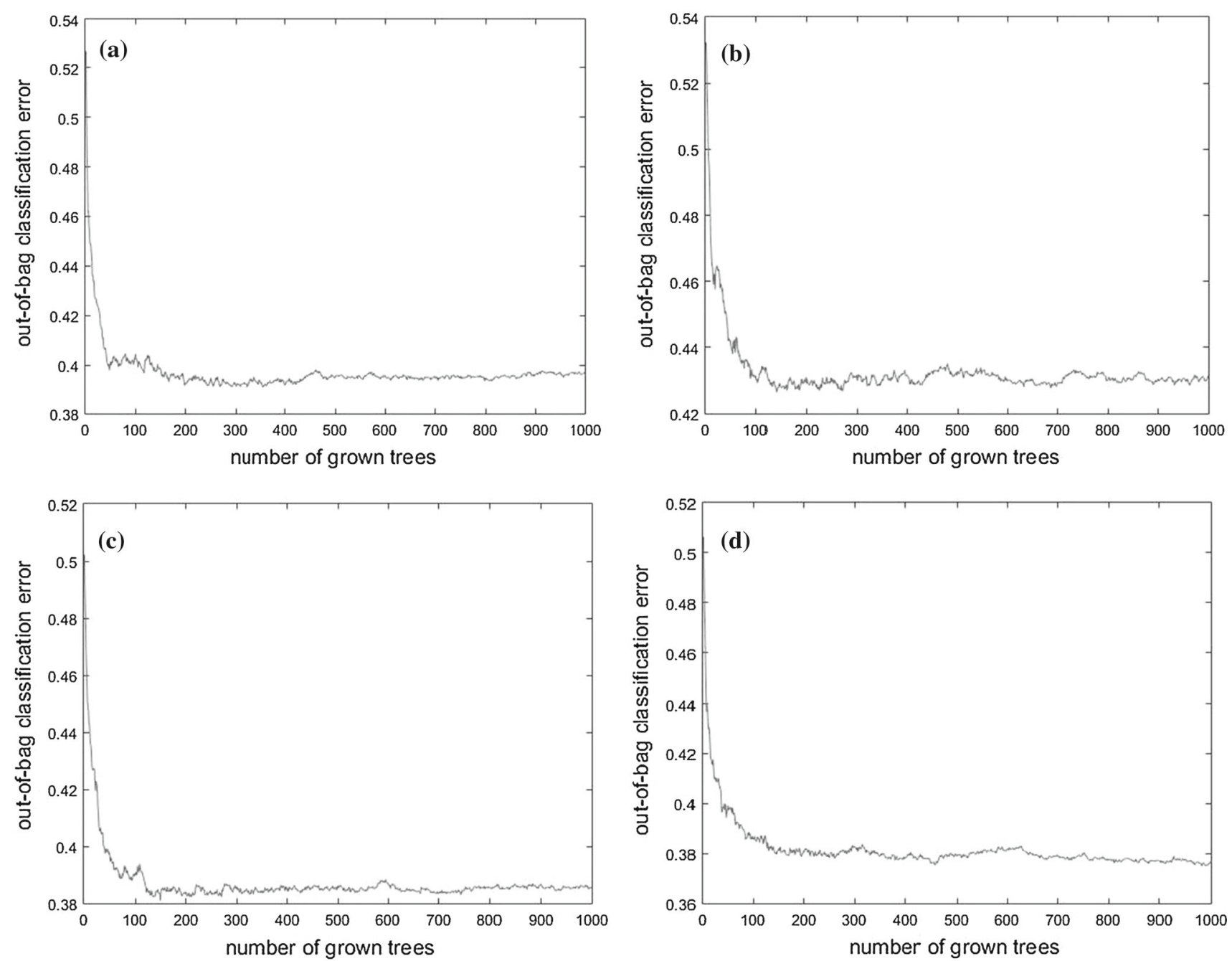

Figure 2. The out-of-bag classification errors produced by the bootstrap aggregated classification tree (BACT) model as a function of the number of grown trees at stations (a) 2815001, (b) 2913001, (c) 2917001, and (d) 3118102.

\subsubsection{Rainfall persistence}

Rainfall persistence is a measure used to evaluate the ability of model in simulating the number of pair of consecutive observed rainy day. It can also be defined as the ratio of number of pair of consecutive rainy days to the total number of rainy days. The rainfall persistence of rainfall occurrence models and their absolute difference compared with the observed rainfall persistence during validation period are presented in table 5. The GLM when compared to NHMM and BACT model, showed the highest absolute difference of rainfall persistence in the range from 0.09 to 0.22 . For the NHMM, the total number of rainy days and two consecutive rainy days, were well simulated with the smallest difference of rainfall persistence in the range from 0.02 to 0.07 . While, the BACT model obtained the absolute difference of rainfall persistence in the range of 0.06 to 0.19 .

The main reason of NHMM having the ability to simulate the observed rainfall persistence is because of the second assumption made during the training of model. First order Markov chain model was used to define the transition probabilities between the hidden states, in which they were not only depending on the set of predictors on a specific day, but also consider the state condition of previous day. However, GLM worked with the approach of logistic regression, and BACT model employed the approach of bagging and classification with if-then logical condition. Both models made no assumption on the condition of rainfall occurrence state on the previous day unlike NHMM. Therefore, the second assumption made in NHMM has been justified with its capability 

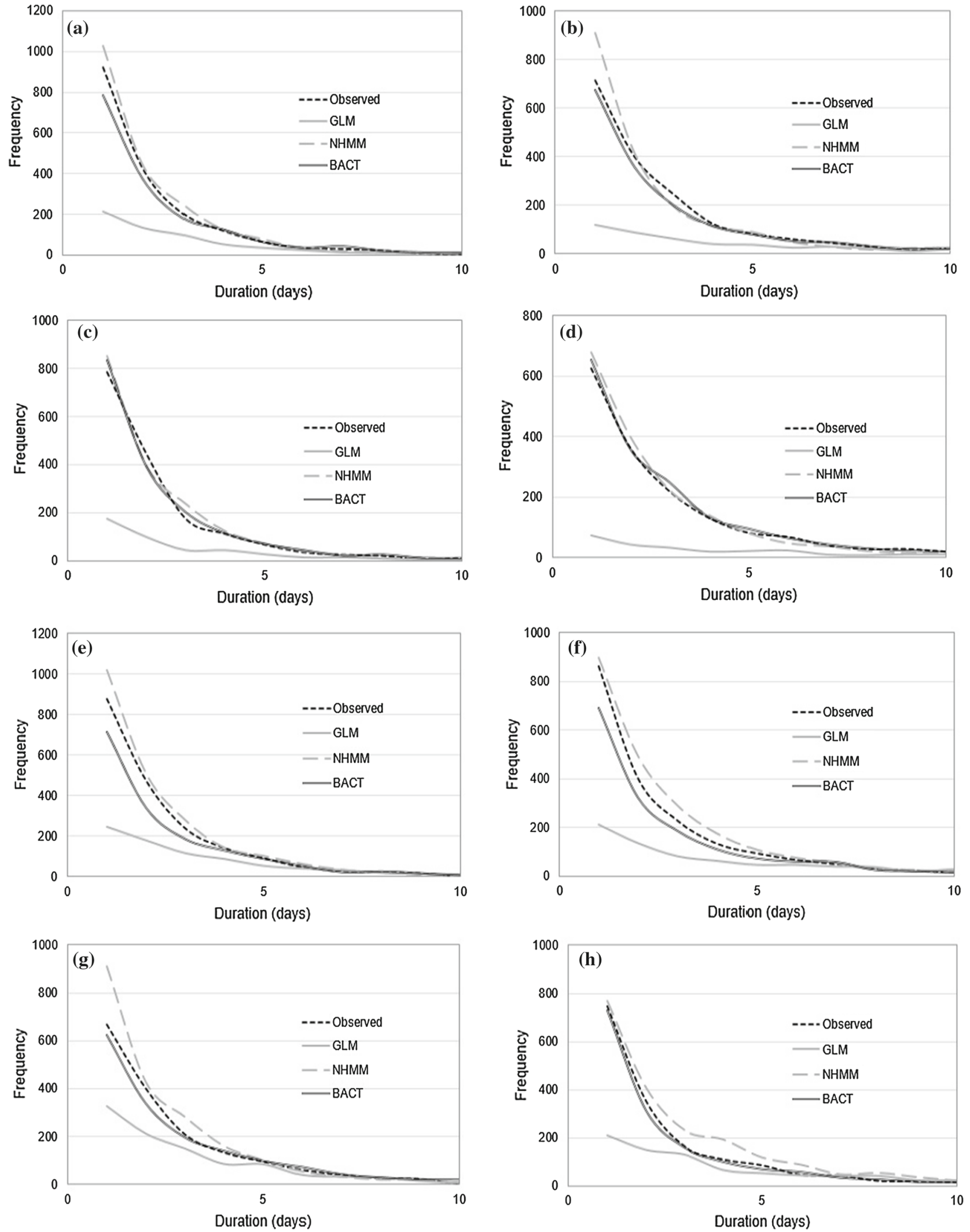

Figure 3. The spell lengths distribution of observed and rainfall occurrence models, the generalized linear model (GLM), the non-homogeneous hidden Markov model (NHMM) and the bootstrap aggregated classification tree (BACT) model. Distribution of (a) wet-spell length at station 2815001, (b) dry-spell length at station 2815001, (c) wet-spell length at station 2913001, (d) dry-spell length at station 2913001, (e) wet-spell length at station 2917001, (f) dry-spell length at station 2917001, (g) wet-spell length at station 3118102, and (h) dry-spell length at station 3118102. 
Table 5. Performance of the generalized linear model (GLM), the non-homogeneous hidden Markov model (NHMM) and the bootstrap aggregated classification tree (BACT) model, in terms of their rainfall persistence and absolute difference with observed rainfall persistence for validation period (1976-2005).

\begin{tabular}{|c|c|c|c|c|}
\hline \multirow[b]{2}{*}{ Station } & \multirow[b]{2}{*}{ Model } & \multicolumn{2}{|c|}{ Rainfall persistence } & \multirow{2}{*}{$\begin{array}{l}\text { Absolute } \\
\text { difference }\end{array}$} \\
\hline & & Observed & Simulated & \\
\hline \multirow[t]{3}{*}{2815001} & GLM & 0.51 & 0.73 & 0.22 \\
\hline & NHMM & 0.51 & 0.58 & 0.07 \\
\hline & $\mathrm{BACT}$ & 0.51 & 0.70 & 0.19 \\
\hline \multirow[t]{3}{*}{2913001} & GLM & 0.55 & 0.69 & 0.14 \\
\hline & NHMM & 0.55 & 0.53 & 0.02 \\
\hline & $\mathrm{BACT}$ & 0.55 & 0.62 & 0.06 \\
\hline \multirow[t]{3}{*}{2917001} & GLM & 0.62 & 0.81 & 0.19 \\
\hline & NHMM & 0.62 & 0.56 & 0.05 \\
\hline & BACT & 0.62 & 0.78 & 0.16 \\
\hline \multirow[t]{3}{*}{3118102} & GLM & 0.63 & 0.72 & 0.09 \\
\hline & NHMM & 0.63 & 0.59 & 0.03 \\
\hline & $\mathrm{BACT}$ & 0.63 & 0.71 & 0.08 \\
\hline
\end{tabular}

of simulating the number of two consecutive rainy days similar to the observed value. Thus, it produced smaller difference of rainfall persistence when compared to GLM and BACT model respectively, at each station.

\subsubsection{Spell lengths distribution}

The distribution of wet- and dry-spell lengths was used to evaluate the performance of rainfall occurrence models at every station during the whole study period (1976-2005). As illustrated in figure 3 , the distribution of wet- and dry-spell lengths was well captured by both the NHMM and BACT model. The spell lengths distribution of both models showed similar trend with the observed dataset by following the approximately geometric distribution. However, NHMM tended to over-predict both spells length at each station, especially a significant difference of wet spell length at stations 2917001 (figure 3e) and 3118102 (figure 3g), and of dry spell length at station 2815001 (figure 3b). In contrast, the BACT model showed the reasonably good simulation results at each station, except for the slight underestimation of both spell lengths at station 2917001 (figure 3e, f).

Compared to NHMM and BACT model, the GLM also tended to follow the geometric distribution, but it showed an obvious underestimation of frequency for the low spell durations ( $<5$ days) at each station, as illustrated in figure 3. Thus, both the NHMM and BACT model showed the better prediction ability than GLM in simulating the observed spell lengths distribution at each station, by considering the huge difference obtained from GLM in comparison with the observed spell lengths distribution.

\subsubsection{Matching}

The performance of rainfall occurrence models were compared and evaluated based on their probability of detection (POD), false alarm rate (FAR), critical success index (CSI), Heidke skill score (HSS) and Peirce skill score (PSS). The ability of a good rainfall occurrence model is to simulate the observed rainfall occurrence as accurate as possible. Thereafter, the rainfall amount values are generated by a rainfall prediction model conditional on the simulated wet days. Both indices of POD and CSI measure the ability of a model to simulate the wet days match with the observed wet days. The higher the index value, the better is the performance of a model.

Based on the results of POD and CSI indices in table 6 , the BACT model performed better than GLM and NHMM during validation period; with the indices within the range of 0.51-0.65 and 0.29 0.44, respectively. The NHMM obtained the POD and CSI indices smaller than 0.5 and 0.3 , respectively, at each station. While, the GLM is capable of obtaining the POD and CSI indices in the range of $0.22-0.54$ and $0.18-0.40$, respectively. The indices of CSI are smaller than those of the POD for the fact that CSI included the number of events, which are predicted but actually not occurred, in their computations. Based on the result of CSI indices, it showed that the NHMM was less capable of generating the wet days matched with the observed wet days, but tended to generate the false alarm of wet days. Hence, the NHMM obtained the smaller CSI indices than BACT model at each station.

In general, FAR should be used in conjunction with POD and it is used to measure the probability of wet day which was simulated by a model, but in reality the rain actually did not occur on that day. Based on the results in table 6, the GLM exhibited better performance with the lowest FAR index (in the range of $0.38-0.52$ ) at each station during the validation period, followed by the BACT model and then the NHMM. The BACT model obtained 
Table 6. Probability of detection (POD), critical success index (CSI) and false alarm rate (FAR) of rainfall occurrence models (generalized linear model (GLM), non-homogeneous hidden Markov model (NHMM) and bootstrap aggregated classification tree (BACT)) for both the calibration period (1976-1995) and the validation period (1976-2005).

\begin{tabular}{|c|c|c|c|c|c|c|c|}
\hline \multirow[b]{2}{*}{ Station } & \multirow[b]{2}{*}{ Model } & \multicolumn{2}{|c|}{ POD } & \multicolumn{2}{|c|}{ CSI } & \multicolumn{2}{|c|}{ FAR } \\
\hline & & Calibration & Validation & Calibration & Validation & Calibration & Validation \\
\hline \multirow[t]{3}{*}{2815001} & GLM & 0.28 & 0.35 & 0.23 & 0.25 & 0.43 & 0.52 \\
\hline & NHMM & 0.38 & 0.40 & 0.23 & 0.23 & 0.62 & 0.64 \\
\hline & $\mathrm{BACT}$ & 0.98 & 0.60 & 0.91 & 0.34 & 0.07 & 0.56 \\
\hline \multirow[t]{3}{*}{2913001} & GLM & 0.17 & 0.22 & 0.15 & 0.18 & 0.42 & 0.51 \\
\hline & NHMM & 0.38 & 0.35 & 0.23 & 0.21 & 0.63 & 0.66 \\
\hline & $\mathrm{BACT}$ & 0.97 & 0.51 & 0.87 & 0.29 & 0.11 & 0.6 \\
\hline \multirow[t]{3}{*}{2917001} & GLM & 0.51 & 0.54 & 0.38 & 0.40 & 0.40 & 0.38 \\
\hline & NHMM & 0.48 & 0.44 & 0.31 & 0.29 & 0.54 & 0.54 \\
\hline & BACT & 0.96 & 0.64 & 0.89 & 0.44 & 0.08 & 0.41 \\
\hline \multirow[t]{3}{*}{3118102} & GLM & 0.43 & 0.45 & 0.34 & 0.34 & 0.40 & 0.41 \\
\hline & NHMM & 0.46 & 0.44 & 0.29 & 0.29 & 0.57 & 0.55 \\
\hline & $\mathrm{BACT}$ & 0.97 & 0.65 & 0.92 & 0.43 & 0.06 & 0.43 \\
\hline
\end{tabular}

Table 7. Heidke skill scores (HSS) and Peirce skill scores (PSS) of rainfall occurrence models (generalized linear model (GLM), non-homogeneous hidden Markov model (NHMM) and bootstrap aggregated classification tree (BACT) model) for both the calibration period (1976-1995) and the validation period (1976-2005).

\begin{tabular}{|c|c|c|c|c|c|}
\hline \multirow[b]{2}{*}{ Station } & \multirow[b]{2}{*}{ Model } & \multicolumn{2}{|c|}{ HSS } & \multicolumn{2}{|c|}{ PSS } \\
\hline & & Calibration & Validation & Calibration & Validation \\
\hline \multirow[t]{3}{*}{2815001} & GLM & 0.17 & 0.16 & 0.15 & 0.15 \\
\hline & NHMM & 0.00 & 0.02 & 0.00 & 0.02 \\
\hline & $\mathrm{BACT}$ & 0.92 & 0.18 & 0.94 & 0.20 \\
\hline \multirow[t]{3}{*}{2913001} & GLM & 0.12 & 0.12 & 0.10 & 0.10 \\
\hline & NHMM & 0.01 & 0.02 & 0.01 & 0.02 \\
\hline & BACT & 0.89 & 0.13 & 0.91 & 0.14 \\
\hline \multirow[t]{3}{*}{2917001} & GLM & 0.25 & 0.25 & 0.24 & 0.25 \\
\hline & NHMM & 0.03 & -0.02 & 0.03 & -0.02 \\
\hline & BACT & 0.89 & 0.25 & 0.89 & 0.26 \\
\hline \multirow[t]{3}{*}{3118102} & GLM & 0.23 & 0.21 & 0.22 & 0.20 \\
\hline & NHMM & 0.03 & 0.00 & 0.03 & 0.00 \\
\hline & BACT & 0.93 & 0.25 & 0.93 & 0.25 \\
\hline
\end{tabular}

the FAR indices in the range of $0.41-0.60$, but the NHMM showed the FAR greater than 0.53 at each station. Hence, it can be concluded that BACT model has the tendency of over-predicting the number of wet days, whence it exhibited higher indices of FAR than GLM but lower than the NHMM, during the validation period and at each station.

Apart from the above, the Heidke skill score (HSS) and Peirce skill score (PSS) were used to summarize the forecasting performance of a model by determining the accuracy of forecast compared to the unskilled reference forecasts (random chance, persistence, or climatology). A forecast with HSS index greater than 0.15 can be treated as reasonably good forecast (Kannan and Ghosh 2011). Based on the results of HSS and PSS indices in table 7, the forecast of NHMM is equivalent to an unskilled random forecast as it obtained 
Table 8. Bayesian information criterion (BIC) scores of deltagamma, delta-exponential with one component and delta-exponential with two component models as a function of different hidden states number at each station.

\begin{tabular}{lcccc}
\hline \multirow{2}{*}{ Station } & \multirow{2}{*}{$\begin{array}{l}\text { Hidden } \\
\text { State }\end{array}$} & Gamma & 1-Exponential & 2-Exponential \\
\cline { 3 - 5 } 2815001 & 1 & 27459.5 & 27611.11 & 27308.07 \\
& 2 & 26609.99 & 26721.29 & 26512.9 \\
2913001 & 3 & $\mathbf{2 4 5 9 4 . 9 5}$ & 26391.13 & 26536.13 \\
& 1 & 28051.76 & 28209.89 & 27938.18 \\
2917001 & 2 & 25275.45 & 27167.13 & 26989.04 \\
& 3 & $\mathbf{2 4 7 6 2 . 6}$ & 26776.24 & 26798.62 \\
3118102 & 1 & 32668.89 & 32894.99 & 32482.79 \\
& 2 & $\mathbf{2 9 1 5 9 . 3 3}$ & 31707.53 & 31653.24 \\
& 2 & 32481.01 & 32823.77 & 32231.69 \\
& 2 & $\mathbf{2 8 5 9 0 . 0 8}$ & 31199.44 & 30876.49 \\
\hline
\end{tabular}

Note: Bold values indicate the minimum BIC score at each station.

both skill scores nearly equal to zero at each station. Except for station 2913001, both the GLM and BACT model showed reasonably good forecast with their skill scores greater than 0.15 at each station during calibration and validation periods. However, the GLM still performed less well than BACT model with their smaller skill score indices during calibration and validation periods. Therefore, the results in table 6 was supported by both HSS and PSS indices in table 7. It can be concluded that the BACT model exhibited better prediction ability than both the GLM and NHMM, and provided higher accuracy in matching the number of observed wet days with slight overestimation over the GLM.

\section{Conclusions}

The first goal of this study is to simulate the observed daily rainfall occurrence in tropical Langat River catchment using the generalized linear model (GLM), the non-homogeneous hidden Markov model (NHMM) and the bootstrap aggregated classification tree (BACT) model. There were two major challenges in this study during the process of developing the NHMM and BACT model. For the determination of optimum number of hidden states in NHMM, it was achieved based on the computed BIC score of each state. A three-state NHMM was chosen for stations 2815001 and 2913001, while a two-state NHMM was found to be adequately used to describe the observed rainfall occurrence at stations 2917001 and 3118102. The major challenge of BACT model in determining the optimum number of grown tree, was overcome by using the prediction error generated from out-of-bag samples. Hence, there was 150 trees grown in the BACT model for this study as the out-of-bag classification error was stabilized and no significant reduction was detected after 150 trees.

The advantage of using NHMM is the application of first-order Markov chain model with the good skill in capturing the weather persistence. Compared to the NHMM, both the GLM and BACT model showed simplicity in modelling the rainfall occurrence using the algorithm of logistic regression and random forest without the application of any hidden state. The main advantages of using logistic regression in GLM are that the predictors do not have to be normally distributed and the output can be explained in terms of probability. For the BACT model, the random forest algorithm was employed to improve the prediction of single classification tree by reducing the variance and increasing the stability of model as compared to single classification tree.

The second goal of this study is to evaluate and select the appropriate rainfall occurrence model based on their performance in capturing the observed daily rainfall occurrence. As predicted, the NHMM exhibited the best performance with the smallest absolute difference of rainfall persistence, followed by the BACT model and GLM. The main reason for the NHMM outperforming the other models is due to the application of firstorder Markov chain model, which consider the state condition of previous day. Both the NHMM and the BACT model are better than the GLM in 
simulating the observed wet- and dry-spell lengths, at each station.

Further to the above, the performance of trained rainfall occurrence models were also evaluated using a $2 \times 2$ contingency table, which can precisely compare the matching between simulated and observed rainfall occurrence series. The BACT model outperformed the GLM and the NHMM with the highest POD and CSI indices. However, the BACT model also slightly over-predicted the number of wet days with the FAR indices higher than GLM, but lower than NHMM. For the comparison in terms of skill score, the skill of NHMM is equivalent to an unskilled random forecast with the HSS and PSS indices nearly equal to zero, at each station. The BACT model is associated with the higher value of indices than the GLM. In a nutshell, the BACT model was selected and recommended as the appropriate daily rainfall occurrence simulation model for the tropical Langat River catchment. The limitation of the selected BACT model was its tendency of over-predicting the number of observed wet days in this study. Hence, the longer study period is recommended in the future research in order to improve the performance of BACT model in simulating the observed rainfall occurrence series, especially to reduce the over-prediction of the number of wet days during validation period.

The future direction should focus on the study of artificial intelligence (AI). In this study, the AI-based model (BACT model) was found to be able to outperform the Markov-chain model (non-homogeneous hidden Markov model) in downscaling the observed rainfall series. Besides, the observed rainfall series and a combination of different variables with the large-scale reanalysis datasets are involved during the application of rainfall downscaling. The convergence of big data is believed to be more important in future research, especially large volume of data is required to achieve the high accuracy of rainfall downscaling. The AI-based model, which can work with big data, will definitely become preferable over the use of conventional model in future study.

\section{Acknowledgements}

The authors would like to express their sincere appreciations to the Universiti Tunku Abdul Rahman, Bandar Sungai Long, Cheras, 43000 Kajang, Selangor, Malaysia for funding this study.

\section{References}

Ahmed K, Shahid S, Haroon S B and Wang X 2015 Multilayer perceptron neural network for downscaling rainfall in arid region: A case study of Baluchistan, Pakistan; J. Earth Syst. Sci. 124(6) 1325-1341.

Beckmann B R and AdriBuishand T 2002 Statistical downscaling relationships for precipitation in the Netherlands and North Germany; Int. J. Climatol. 22(1) 15-32, https://doi.org/10.1002/joc.718.

Breiman L 2001 Random forests; Mach. Learn. 45 5-32, https://doi.org/10.1023/A:1010933404324.

Buishand T A, Shabalova M V and Brandsma T 2004 On the choice of the temporal aggregation level for statistical downscaling of precipitation; J. Clim. 17(9) 1816-1827.

Chau K W and Wu C L 2010 A hybrid model coupled with singular spectrum analysis for daily rainfall prediction; J. Hydroinform. 12(4) 458-473, https://doi.org/10.2166/ hydro.2010.032.

Fealy R and Sweeney J 2007 Statistical downscaling of precipitation for a selection of sites in Ireland employing a generalised linear modelling approach; Int. J. Climatol. 27(15) 2083-2094, https://doi.org/10.1002/joc.1506.

Fu G, Charles S P and Kirshner S 2013 Daily rainfall projections from general circulation models with a downscaling non-homogeneous hidden Markov model (NHMM) for south-eastern Australia; Hydrol. Process. 27(25) 3663 3673, https://doi.org/10.1002/hyp.9483.

Gaitan C F, Hsieh W W and Cannon A J 2014 Comparison of statistically downscaled precipitation in terms of future climate indices and daily variability for southern Ontario and Quebec, Canada; Clim. Dyn. 43(12) 32013217, https://doi.org/10.1007/s00382-014-2098-4.

Greene A M, Robertson A W, Smyth P and Triglia S 2011 Downscaling projections of Indian monsoon rainfall using a non-homogeneous hidden Markov model; Quart. J. Roy. Meteorol. Soc. 137 347-359, https://doi.org/10.1002/qj. 788.

He X, Chaney N W, Schleiss M and Sheffield J 2016 Spatial downscaling of precipitation using adaptable random forests; Water Resour. Res. 52 8217-8237, https://doi. org/10.1002/2016WR019034.

Jing W, Yang Y, Yue X and Zhao X 2016 A comparison of different regression algorithms for downscaling monthly satellite-based precipitation over north China; Remote Sens. 8(10) 835-851, https://doi.org/10.3390/ rs8100835.

Kannan S and Ghosh S 2011 Prediction of daily rainfall state in a river basin using statistical downscaling from GCM output; Stoch. Environ. Res. Risk Assess. 25(4) 457-474, https://doi.org/10.1007/s00477-010-0415-y.

Kannan S and Ghosh S 2013 A nonparametric kernel regression model for downscaling multisite daily precipitation in the Mahanadi basin; Water Resour. Res. 49(3) 1360 1385, https://doi.org/10.1002/wrcr.20118.

Kenabatho P K, Mclntyre N R, Chandler R E and Wheater H S 2012 Stochastic simulation of rainfall in semi-arid Limpopo basin, Botswana; Int. J. Climatol. 32(7) 11131127, https://doi.org/10.1002/joc.2323.

Kioutsioukis I, Melas D and Zanis P 2008 Statistical downscaling of daily precipitation over Greece; Int. J. Climatol. 28(5) 679-691, https://doi.org/10.1002/joc.1557. 
Lee T C, Chan K Y, Chan H S and Kok M H 2011 Projections of extreme rainfall in Hong Kong in the 21st century; Acta Meteorol. Sin. 25(6) 691-709.

Liaw A and Wiener M 2002 Classification and regression by random forest; $R$. News $\mathbf{2 ( 3 )} 18-22$.

Liu W, Fu G, Liu C and Charles S P 2013 A comparison of three multi-site statistical downscaling models for daily rainfall in the North China Plain; Theor. Appl. Climatol. 111(3-4) 585-600, https://doi.org/10.1007/ s00704-012-0692-0.

Liu Z, Xu Z, Charles SP, Fu G and Liu L 2011 Evaluation of two statistical downscaling models for daily precipitation over an arid basin in China; Int. J. Climatol. 31(13) 2006-2020, https://doi.org/10.1002/joc.2211.

Mandal S, Srivastav R K and Simonovic S P 2016 Use of beta regression for statistical downscaling of precipitation in the Campbell River basin, British Columbia, Canada; J. Hydrol. 538 49-62, https://doi.org/10.1016/j.jhydrol. 2016.04.009.

Mares C, Mares I, Huebener H, Mihailescu M, Cubasch U and Stanciu P 2014 A hidden Markov model applied to the daily spring precipitation over the Danube Basin; Adv. Meteorol. 2014 ID 237247.

Memarian H, Balasundram S K, Abbaspour K C, Talib J B, Christopher Teh B S and Sood A M 2014 SWAT-based hydrological modelling of tropical-use scenarios; Hydrol. Sci. J. 59(10) 1808-1829.

Ng J L, Aziz S A, Huang Y F, Wayayok A and Rowshon M K 2017 Generation of a stochastic precipitation model for the tropical climate; Theor. Appl. Climatol., https://doi. org/10.1007/s00704-017-2202-x.

Pineda A N and Willems P 2016 Multisite downscaling of seasonal predictions to daily rainfall characteristics over Pacific-Andean River Basins in Eucadoe and Peru using a nonhomogeneous hidden Markov model; J. Hydrolmeteorol. 17(2) 481-498.

Robertson A W, Kirshner S and Smyth P 2004 Downscaling of daily rainfall occurrence over northeast Brazil using a hidden Markov model; J Climate 17(22) 4407-4424, https://doi.org/10.1175/JCLI-3216.1.
Robertson A W, Moron V and Swarinoto Y 2009 Seasonal predictability of daily rainfall statistics over Indramayu district, Indonesia; Int. J. Climatol. 29(10) 1449-1462, https://doi.org/10.1002/joc.1816.

Salvi K, Kannan S and Ghosh S 2013 High-resolution multisite daily rainfall projections in India with statistical downscaling for climate change impacts assessment; $J$. Geophys. Res. Atmos. 118(9) 3557-3578, https://doi. org/10.1002/jgrd.50280.

Saudi A S M, Juahir H, Azid A, Toriman M E, Kamarudin M K A, Saudi M M, Mustafa A D and Amran M A 2015 Flood risk pattern recognition by using environmetric technique: A case study in Langat river basin; J. Teknol. 77(1) 145-152.

Schoof J T and Pryor S C 2001 Downscaling temperature and precipitation: A comparison of regression-based methods and artificial neural networks; Int. J. Climatol. 21(7) 773-790, https://doi.org/10.1002/joc.655.

Shi Y and Song L 2015 Spatial downscaling of monthly TRMM precipitation based on EVI and other geospatial variables over the Tibetan Plateau from 2001 to 2012; Mt. Res. Dev. 35(2) 180-194, https://doi.org/10.1659/ MRD-JOURNAL-D-14-00119.1.

Singh D, Jain S K and Gupta R D 2015 Statistical downscaling and projection of future temperature and precipitation change in middle catchment of Sutlej River Basin, India; J. Earth Syst. Sci. 124(4) 843-860.

Sullivan C A and Huntingford C 2009 Water resources, climate change and human vulnerability; Proceedings of the 18th World IMACS/MODSIM Congress, Cairns, Australia, pp. 3984-3990.

Wilby R L and Dawson C W 2013 The statistical downscaling model: Insights from one decade of application; Int. J. Climatol. 33(7) 1707-1719, https://doi.org/10.1002/joc. 3544.

Wu C L, Chau K W and Fan C 2010 Prediction of rainfall time series using modular artificial neural network coupled with data-preprocessing techniques; J. Hydrol. 389(1-2) 146-167, https://doi.org/10.1016/j.hydrol. 2010.05.040 